\section{Acute respiratory distress syndrome associated with tumor lysis syndrome in a child with acute lymphoblastic leukemia}

\author{
Alessandra Macaluso, ${ }^{1}$ Selene Genova, ${ }^{1}$ \\ Silvio Maringhini, 2 Giancarlo Coffaro, 3 \\ Ottavio Ziino, 4 \\ Paolo D'Angelo4
}

1Pediatric Department and Postgraduate School, University of Palermo; 2Pediatric Nephrology Unit, G. Di Cristina Children's Hospital, Palermo; ${ }^{3 P e d i a t r i c ~ I n t e n s i v e ~}$ Care Unit, G. Di Cristina Children's Hospital, Palermo; 4Pediatric Hematology and Oncology Unit, A.R.N.A.S. Civico, Di Cristina and Benfratelli Hospital, Palermo, Italy

\section{Abstract \\ Tumor lysis syndrome is a serious and dan- gerous complication usually associated with antiblastic treatment in some malignancies characterized by high cell turn-over. Mild or severe electrolyte abnormalities including high serum levels of uric acid, potassium, phosphorus, creatinine, bun and reduction of calcium can be responsible for multi-organ failure, involving mostly kidneys, heart and central nervous system. Renal damage can be followed by acute renal failure, weight gain, progressive liver impairment, overproduction of cytokines, and subsequent maintenance of multi-organ damage. Life-threatening acute respiratory failure associated with tumor lysis syndrome is rare. We describe a child with T- cell acute lymphoblastic leukemia, who devel- oped an unusually dramatic tumor lysis syn- drome, after administration of the first low doses of steroid, that was rapidly associated with severe acute respiratory distress syn- drome. Subsequent clinical course and treat- ment modalities that resulted in the gradual and full recovery of the child are also described.}

\section{Introduction}

Tumor lysis syndrome (TLS) is a very serious and potentially life-threatening event. It is caused by the massive release of nucleic acids, potassium and phosphate into the blood as a result of tumor cell lysis, usually associated with tumor cell death after treatment. Moreover, TLS may be observed before starting the treatment due to spontaneous tumor cell death.1-3 It may present with multiple electrolyte abnormalities including hyperuricemia, hyperphosphatemia, hypocalcemia and hyperkalemia, and can lead to multi-organ dysfunction, affecting kidneys, heart, skeletal muscle and central nervous system. Burkitt lymphoma and T-cell acute lymphoblastic leukemia (ALL) or lymphoblastic lymphoma are most frequently associated with this complication. Comorbidities predisposing to higher risk of developing TLS are elevated pre-treatment serum uric acid level, pre-existing renal damage, tumor infiltration in the kidney, obstructive uropathy, and advanced age. 4

We describe a child with T-ALL, who developed unexpected dramatic TLS, after administration of the first low doses of steroid that was rapidly associated with severe acute respiratory distress syndrome (ARDS).

\section{Case Report}

A previously healthy 7-year-old boy with fever for seven days, headache, ocular pain and bilateral proptosis underwent a blood test that revealed marked leucocytosis, white blood cell count (WBC) 478,000/ $\mu \mathrm{L}$, slight anemia, red blood cells (RBC) 4,340,000/ $\mu \mathrm{L}$, hemoglobin $(\mathrm{Hb}) 10 \mathrm{~g} / \mathrm{dL}$, and mild thrombocytopenia (platelets $79,000 / \mu \mathrm{L}$ ). He was immediately admitted to our Unit and a bone marrow aspirate confirmed the diagnosis of T-ALL. A central venous catheter was inserted, and correct insertion confirmed by chest X-ray, which showed right lung hypodiaphany. Lumbar puncture revealed an absence of lymphoblasts in cerebrospinal fluid. So he was enrolled in the AIEOP (Associazione Italiana di Ematologia ed Oncologia Pediatrica) ALL R2006 protocol. The child underwent supportive treatment including hyper-hydration (glucose and sodium chloride solution 3000 $\mathrm{mL} / \mathrm{sqm} /$ day $)$ with bicarbonate (100 $\mathrm{mmol} / \mathrm{sqm})$, allopurinol $(15 \mathrm{mg} / \mathrm{kg} /$ day in 3 doses); a low dose of prednisone (10 $\mathrm{mg} / \mathrm{sqm} /$ day in 2 doses) was commenced, together with accurate renal function monitoring. On the second day WBC dropped to $127,000 / \mu \mathrm{L}$, and normal renal function parameters persisted. After administration of a total of $30 \mathrm{mg} / \mathrm{sqm}$ of steroid in five doses in about a 36-hour-period, WBC dropped to $32,800 / \mu \mathrm{L}$ and he developed shock (pale and cold skin, small wrists, capillary Refill $>4$ ", AP 78/57 $\mathrm{mmHg}$, CF $132 \mathrm{bpm}$ ). Arterial blood gas value analysis revealed metabolic acidosis $(\mathrm{pH} 7.20$, $\mathrm{PO}_{2} 55 \mathrm{mmHg}, \mathrm{PCO}_{2} 32 \mathrm{mmHg}, \mathrm{HCO}_{3^{-}} 12.5$ $\mathrm{mmol} / \mathrm{L}, \mathrm{BE}-15.5, \mathrm{SatO}_{2} 79.8 \%$ ). Blood tests showed uric acid $6 \mathrm{mg} / \mathrm{dL}$, BUN $124 \mathrm{mg} / \mathrm{dL}$, creatinine $2.9 \mathrm{mg} / \mathrm{dL}$, potassium $6.9 \mathrm{mmol} / \mathrm{L}$, calcium $4.3 \mathrm{mg} / \mathrm{dL}$, phosphorus $26.6 \mathrm{mg} / \mathrm{dL}$, support-
Correspondence: Paolo D’Angelo, U.0. di Oncoematologia Pediatrica, Padiglione Oncologico, Ospedale Civico, Piazza Nicola Leotta 4, 90127 Palermo, Italy.

Tel.: +39.91.666.4142/143; Fax +39.91.666.4127. E-mail: oncoematoped@ospedalecivicopa.org; papagisa@libero.it

Key words: acute respiratory distress syndrome, tumor lysis syndrome, acute lymphoblastic leukemia, childhood.

Acknowledgements: the authors are grateful to Prof. Frank Adamo for language editing, and the parents' Association “A.S.L.T.I.-Liberi di crescere" Onlus for the financial support.

Contributions: AM, SG and PD, manuscript writing and references searching; SM, GC, OZ and PD manuscript reviewing; SM, GC, OZ and PD were equally involved in the clinical management of the patient.

Conflict of interest: the authors declare no potential conflict of interest.

Received for publication: 14 December 2014 Revision received: 24 January 2015.

Accepted for publication: 24 January 2015.

This work is licensed under a Creative Commons Attribution NonCommercial 3.0 License (CC BYNC 3.0).

(C) Copyright A. Macaluso et al., 2015

Licensee PAGEPress, Italy

Pediatric Reports 2015; 7:5760

doi:10.4081/pr.2015.5760

ing the diagnosis of TLS with acute renal failure.

The patient was treated aggressively with intravenous fluids, allopurinol therapy, rasburicase, calcium gluconate, insulin therapy for hyperkaliemia, and diuretics. Due to anemia and thrombocytopenia he also needed red blood cell and platelet transfusions.

The following day he developed respiratory failure. A chest radiograph taken at this time revealed bilateral pulmonary infiltrates consistent with ARDS (Figure 1). Transfusion related acute lung injury (TRALI) was excluded because even though it is clinically indistinguishable from acute respiratory distress syndrome (ARDS), it occurs within 6 hours after transfusion. Unlike ARDS, TRALI is self-limiting, and there is usually clinical improvement within 48-96 hours if prompt respiratory support is provided. 5 In our case significant levels of positive end-expiratory pressure and a high fraction of inspired oxygen (protective ventilation) were needed to maintain adequate systemic oxygenation. Despite medical treatment, hemofiltration (five sessions in three days) was necessary to control persistent hyper- 
kalemia, hyperphophoremia with hypocalcemia, and also to remove circulating inflammatory cytokines (probably related to ARDS). Steroids were continued at the dose of 10 $\mathrm{mg} / \mathrm{sqm} /$ day, in order to obtain an adequate control of leukemic cells count, and antiinflammatory effect. He was intubated for one month and managed with continued mechanical ventilation. There was a gradual improvement of respiratory function, weaning from $\mathrm{O}_{2}$ therapy, and normalization of renal function with reversal of the altered hemodynamic factors. When the clinical condition improved he resumed chemotherapy according to the protocol, and currently he's well in first complete remission, after discontinuation of chemotherapy, 36 months after diagnosis (laboratory findings are available in Supplementary Tables S1-S3).

\section{Discussion}

According to the consensus conference on the management of tumor lysis syndrome, laboratory TLS is defined by the occurrence of two or more of the following serum values before or after anticancer treatment (from three days before to seven days after the start of anticancer treatment): i) uric acid: increase of more than $25 \%$ from baseline, or $476 \mathrm{mmol} / \mathrm{L}$ $(8 \mathrm{mg} / \mathrm{dL})$; ii) potassium: increase of more than $25 \%$ from baseline, or $6.0 \mathrm{mmol} / \mathrm{L}$ (6 $\mathrm{mEq} / \mathrm{L})$; iii) phosphorus: increase of more than $25 \%$ from baseline, or $1.45 \mathrm{mmol} / \mathrm{L}(4.5 \mathrm{mg} / \mathrm{dL})$ in adults, and $2.1 \mathrm{mmol} / \mathrm{L}(6.5 \mathrm{mg} / \mathrm{dL})$ in children; iiii) calcium: decrease of more than $25 \%$ from baseline, or $1.75 \mathrm{mmol} / \mathrm{L}(7 \mathrm{mg} / \mathrm{dL}){ }^{4}$

Clinical TLS is defined by the presence of laboratory TLS and at least one of the following clinical alterations: renal failure (estimated glomerular filtration rate $<60 \mathrm{~mL} / \mathrm{min}$ ), cardiac arrhythmia/sudden death, seizures. Grading of clinical TLS ranges from I to IV and is based on the degree of elevation of serum creatinine, the presence and type of cardiac arrhythmia, and the presence and severity of seizure.

Potential predictors of TLS include laboratory features, such as high initial WBC count, elevated serum LDH or uric acid levels, and clinical indicators of bulky disease, such as the presence of a mediastinal mass on chest radiographs, hepatomegaly (defined as a palpable liver $3 \mathrm{~cm}$ below the right costal margin), and splenomegaly (defined as a palpable spleen $2 \mathrm{~cm}$ below the left costal margin), as assessed by the physical examination on admission, pre-existing dehydration, oliguria, or renal failure and malignancies with high sensitivity to chemotherapy. 6

Drugs for TLS prophylaxis include allopurinol, which blocks the activity of the liver enzyme xanthine oxidase, and thereby decreases the risk of uric acid crystallization in the kidneys, 7,8 and rasburicase, a recombinant urate oxidase enzyme, which converts existing uric acid to allantoin, which is 5 to 10 times more soluble in urine than uric acid. Effective treatment strategies that have been reported useful in TLS include vigorous hydration, allopurinol or rasburicase administration. Renal replacement therapy occasionally is needed. Indications for the start-up of renal replacement therapy in TLS include persistent hyperkalemia, severe metabolic acidosis, volume overload unresponsive to diuretic therapy, and overt uremic symptoms.

In our patient hypovolemic shock developed subsequently to TLS and acute renal failure; later severe ARDS began as a probable consequence of overproduction of cytokines. Despite medical treatment, continuous renal replacement therapy was necessary and after that only respiratory function gradually improved. To our knowledge, only one case of a 26 -year-old woman with ARDS in TLS is reported in literature. ${ }^{9}$ The risk of developing ARDS in the setting of TLS may depend on the degree of cell lysis, the speed of release of mediators into pulmonary circulation, and the susceptibility of the alveolar-capillary membrane to injury.

The pathogenesis of ARDS in the setting of TLS remains unclear. It could be related to the release of cytokines or other active mediators, and not to the serum electrolyte abnormalities. ${ }^{10}$ It has been hypothesized that an excessive inflammatory response could be down-regulated with continuous renal replacement therapies (CRRT), by non specific extracorporeal removal of cytokines, and other mediators. ${ }^{11}$ Absorption appears to be the predominant mechanism of mediator elimination. In addition, the eventual elimination of inflammatory mediators, fluid removal with reduction of extravascular lung water (EVLW), is a second mechanism by which hemofiltration may be beneficial in ARDS. However, the observed hemodynamic improvement can at least partially be attributed to a reduction of body temperature, and the evidence for clinically important removal of pro-inflammatory cytokines remains limited. ${ }^{12,13}$ CRRT-induced hypothermia can be used in patients with ARDS in order to reduce $\mathrm{CO}_{2}$ production. The decreased ventilator requirement reduces the risk of ventilatorinduced lung injury. Reduced $\mathrm{CO}_{2}$ production combined with the alkalinizing effect of bicarbonate in the replacement solution facilitates the institution of permissive hypercapnia. An advantage of this procedure is the ability to remove large volumes of fluid while avoiding the hypotensive episodes caused by intermittent hemodialysis, so it is indicated for managing patients with acute renal failure who are hemodynamically unstable.

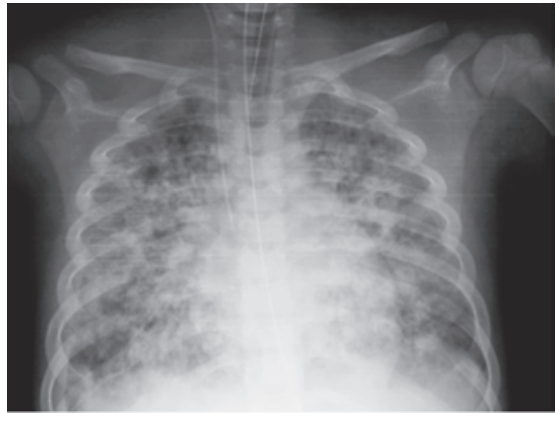

Figure 1. Chest radiograph shows multiple bilateral pulmonary infiltrates suggesting an acute respiratory distress syndrome.

\section{Conclusions}

Tumor lysis syndrome (TLS) is a very serious and potentially life-threatening event, that can lead to severe multi-organ dysfunction. Intensive supportive treatment, including hyper-hydration and allopurinol could be not enough in some malignancies characterized by a rapid cellular turn over and the administration of rasburicase represent the first choice prophylaxis, that must be started as soon as possible. ARDS can be an unusual complication of TLS, probably related to overproduction of cytokines, also in spite of steroid treatment. A prompt hemofiltration may be an effective measure for both clinical conditions, clearing the excess of circulating cytokines and supplying renal function.

\section{References}

1. Wilson FP, Berns JS. Tumor lysis syndrome: new challenges and recent advances. Adv Chronic Kidney Dis 2014; 21:18-26.

2. McBride A, Westervelt P. Recognizing and managing the expanded risk of tumor lysis syndrome in hematologic and solid malignancies. J Hematol Oncol 2012;5:75.

3. Yo HA, Hyoung JK, Hee YS, et al. Tumor lysis syndrome in children: experience of last decade. Hematol Oncol 2011;29:196201.

4. Tosi P, Barosi G, Lazzaro C, et al. Consensus conference on the management of tumor lysis syndrome. Haematologica 2008;93:1877-85.

5. Sayah DM, Looney MR, Toy P. Transfusion reactions: newer concepts on the pathophysiology, incidence, treatment, and pre- 
vention of transfusion-related acute lung injury. Crit Care Clin 2012;28:363-72.

6. Truong TH, Beyene J, Hitzler J, et al. Features at presentation predict children with acute lymphoblastic leukemia at low risk for tumor lysis syndrome. Cancer 2007;110:1832-9.

7. De Conti RC, Calabresi P. Use of allopurinol for prevention and control of hyperuricemia in patients with neoplastic disease. N Engl J Med 1996;274:481-6.

8. Hande KR, Hixon CV, Chabner BA. Post chemotherapy purine excretion in patients receving allopurinol. Cancer Res 1981;41:2273-9.

9. Marenco JP, Nervi A, White AC. ARDS associated with tumor lysis syndrome in a patient with non-Hodgkin's lymphoma. Chest 1998;113:550-2.

10. Saxon RR, Klein JS, Bar MH, et al. Pathogenesis of pulmonary edema during interleukin-2 therapy: correlation of chest radiograph and clinical findings in 54 patients. Am J Radiol 1991;156:281-5.

11. Grootendorst AF. The potential role of hemofiltration in the treatment of patients with septic shock and multiple organ dysfunction syndrome. Adv Renal Replace Ther 1994;1:176-84.

12. Schetz M. Non-renal indications for continuous renal replacement therapy. Kidney Int 1999;72:88-94.

13. Choi KA, Jung EL, Yoon-Goo K, et al. Efficacy of continuous veno-venous hemofiltration with chemotherapy in patients with Burkitt lymphoma and leukemia at high risk of tumor lysis syndrome. Ann Hematol 2009;88:639-45. 